\title{
Shielded cantilever with on-chip interferometer circuit for THz scanning probe impedance microscopy
}

Cite as: Rev. Sci. Instrum. 90, 113701 (2019); https://doi.org/10.1063/1.5116801

Submitted: 27 June 2019 . Accepted: 15 October 2019 . Published Online: 05 November 2019

Matvey Finkel (D), Holger Thierschmann (D), Allard J. Katan (D), Marc P. Westig (D), Marco Spirito, and Teun M. Klapwijk (D)

\section{ARTICLES YOU MAY BE INTERESTED IN}

Signal reversal in Kelvin-probe force microscopy

Review of Scientific Instruments 90, 113703 (2019); https://doi.org/10.1063/1.5118357

Single-pass non-destructive electronic detection of charged particles

Review of Scientific Instruments 90, 113301 (2019); https://doi.org/10.1063/1.5110988

Low current heaterless hollow cathode neutralizer for plasma propulsion-Development overview

Review of Scientific Instruments 90, 113303 (2019); https://doi.org/10.1063/1.5097599

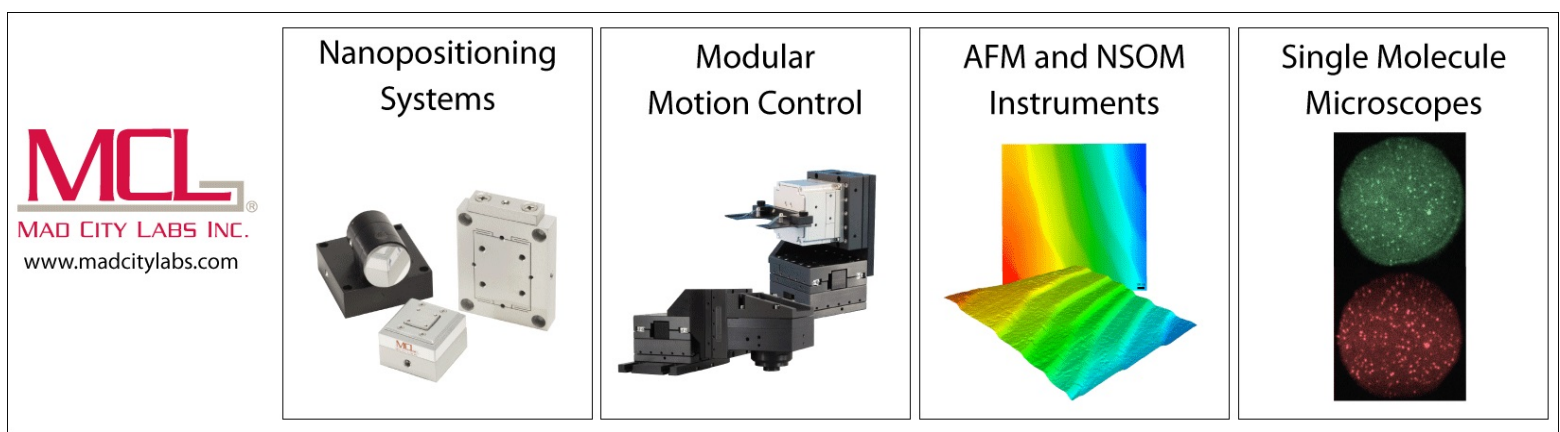




\title{
Shielded cantilever with on-chip interferometer circuit for THz scanning probe impedance microscopy
}

\author{
Cite as: Rev. Sci. Instrum. 90, 113701 (2019); doi: 10.1063/1.5116801 \\ Submitted: 27 June 2019 - Accepted: 15 October 2019 • \\ Published Online: 5 November 2019

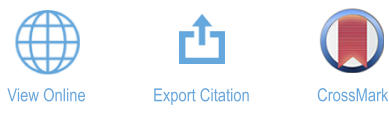

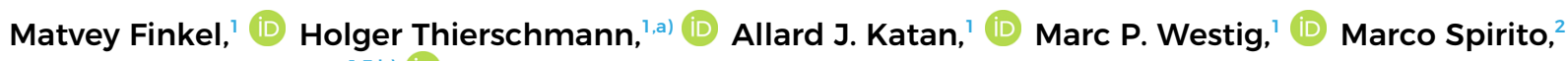
and Teun M. Klapwijk ${ }^{1,3, b)}$ (D)

\author{
AFFILIATIONS \\ ${ }^{\top}$ Kavli Institute of NanoScience, Department of Quantum Nanoscience, Faculty of Applied Sciences, \\ Delft University of Technology, Lorentzweg 1, 2628 CJ Delft, The Netherlands \\ ${ }^{2}$ Department of Microelectronics, Faculty of Electrical Engineering, Delft University of Technology, Mekelweg 4, \\ 2629 JA Delft, The Netherlands \\ ${ }^{3}$ Physics Department, Moscow State Pedagogical University, Moscow 119991, Russia \\ a)hthiersch@gmail.com \\ b)t.m.klapwijk@tudelft.nl
}

\begin{abstract}
We have realized a microstrip based terahertz $(\mathrm{THz})$ near field cantilever that enables quantitative measurements of the impedance of the probe tip at $\mathrm{THz}$ frequencies $(0.3 \mathrm{THz})$. A key feature is the on-chip balanced hybrid coupler that serves as an interferometer for passive signal cancellation to increase the readout circuit sensitivity despite extreme impedance mismatch at the tip. We observe distinct changes in the reflection coefficient of the tip when brought into contact with different dielectric $\left(\mathrm{Si}, \mathrm{SrTiO}_{3}\right)$ and metallic samples (Au). By comparing finite element simulations, we determine the sensitivity of our $\mathrm{THz}$ probe to be well below $0.25 \mathrm{fF}$. The cantilever further allows for topography imaging in a conventional atomic force microscope mode. Our THz cantilever removes several critical technology challenges and thus enables a shielded cantilever based $\mathrm{THz}$ near field microscope.
\end{abstract}

Published under license by AIP Publishing. https://doi.org/10.1063/1.5116801

\section{INTRODUCTION}

In condensed matter research, there is a strong desire for new experimental tools that can measure the local electrical properties of materials and buried layers at high frequencies and with high spatial resolution. ${ }^{1}$ For this goal, in recent years, a variety of powerful scanning probe techniques have emerged, which cover different parts of the electromagnetic spectrum: on the one hand, scanning near-field optical microscopy (SNOM) has enabled imaging with infrared and far-infrared $^{2-4}$ frequencies down to a few terahertz (THz) by utilizing the optical toolbox, i.e., free-space radiation, and lasers and fiber technology; on the other hand, at gigahertz frequencies (typically 1-20 GHz), coaxial probes and shielded cantilevers have made possible quantitative local imaging by making use of commercially available microwave electronics for high performance signal processing (scanning microwave impedance microscopy, SMIM)..$^{5-12}$ The frequency band in between, however, ranging approximately from $100 \mathrm{GHz}$ to a few $\mathrm{THz}$ (also referred to as sub-millimeter waves), is a technological challenge. In the field of astronomy detection, major progress has been made in sub-mm technology, for instance in the development of phase preserving instruments based on superconducting tunnel junctions such as for the Herschel Space Telescope, the Atacama Pathfinder Experiment (APEX), and the Atacama Large Millimeter array (ALMA). ${ }^{13,14}$ These advances are being picked up to promote technological progress also in other research fields. In condensed matter physics, this is expected to have a strong impact on measurement instrument development, which will help understanding a variety of important problems, in particular for disordered and unconventional superconductors as well as for the so-called quantum materials where strong electron-electron interactions 
in the $\mathrm{THz}$ energy range give rise to a number of puzzling, unconventional, and often spatially inhomogeneous electrical properties. ${ }^{1,3,15}$ Realizing an experimental tool for probing these properties, however, remains challenging. Only recently, first scattering SNOM measurements below $1 \mathrm{THz}$ have been reported. ${ }^{16}$ In the sub-mm band, on-chip electronic circuits suffer from high losses (at room temperature), which strongly complicates the fabrication of more complex circuitry, required for signal control and processing. An alternative technology, much less prone to losses, consists of quasioptical and metallic waveguide components. However, this technology increases the size of the measurement instrument compared with on-chip circuitry and thus also imposes certain boundaries when more complex signal handling is needed. In order to overcome these technological hurdles, there is ongoing development to combine quasioptical and on-chip electronics in hybrid devices ${ }^{17}$ but also to push the performance of microwave electronics into the sub-millimeter-band. ${ }^{18}$ Picking up on this development, we have recently reported a microstrip (MS) fabrication technology based on PECVD $\mathrm{SiN}_{x}$ that is compatible with thin film membranes. For this technology, losses are sufficiently well controlled at frequencies around $0.3 \mathrm{THz}$ such that the realization of room-temperature $\mathrm{THz}$ on-chip components is feasible. ${ }^{19}$ Here, we use this technology to extend scanning impedance microscopy from microwaves into the $\mathrm{THz}$ frequency range. We present a shielded $\mathrm{THz}$ cantilever suitable for scanning probe microscopy that enables quantitative measurement of the impedance of the cantilever tip at around $0.3 \mathrm{THz}$. A key ingredient is a branchline coupler that is patterned on the cantilever and acts as an interferometer for the $\mathrm{THz}$ signal, thereby providing high sensitivity of the circuit to small impedance changes at the cantilever tip.

First, we will revisit the concept of scanning near field microscopy with shielded cantilevers as it is currently being used in microwave microscopy, and we will identify the key features a $\mathrm{THz}$ cantilever should comprise. We then present the concept of our $\mathrm{THz}$ on-chip interferometer. Finally, we demonstrate how this concept enables impedance measurements with a $\mathrm{THz}$ cantilever that is compatible with conventional atomic force microscopy (AFM).

\section{PRINCIPLE OF SHIELDED CANTILEVER MICROSCOPY}

The principle of scanning near field microscopy with a shielded microstrip (MS) cantilever is illustrated in Fig. 1(a).,20 The cantilever consists of a dielectric membrane of which the bottom side is covered with a thin metal layer, serving as a transmission line ground plane. The signal line of width $w$ is patterned on the top side of the same dielectric. A cross section of the resulting MS transmission line geometry is sketched in Fig. 1(b). Because the high frequency fields are mostly confined within the dielectric, such a transmission line geometry allows for delivering the signal to the cantilever tip in a controlled way, while the ground plane screens the environment and prevents radiation losses. At the end of the cantilever, the signal line terminates in a metallic tip. When a high frequency tone is launched to the MS, the tip acts as a capacitive termination, reflecting the signal back into the cantilever. This is quantified by the reflection coefficient $\Gamma$, which is given by the mismatch between the generally complex valued tip impedance $Z$ and the characteristic MS

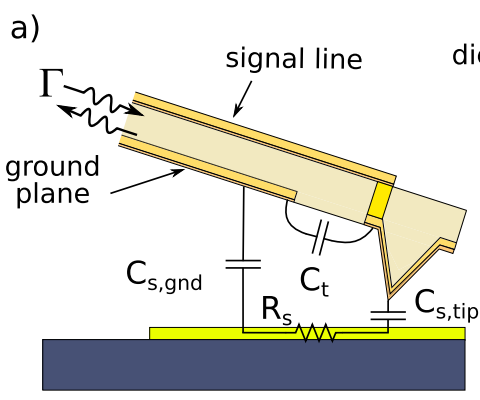

b)

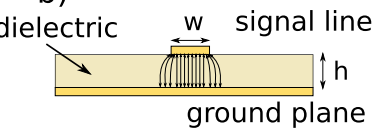

c)

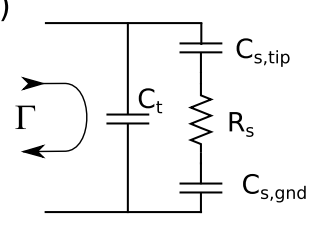

arm 2 to

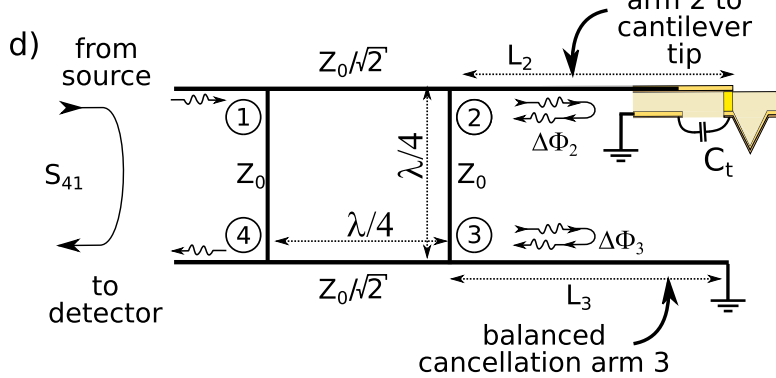

FIG. 1. (a) Sketch of a shielded cantilever and the corresponding lumped element circuit for measuring the complex reflection coefficient $\Gamma$ of the cantilever tip. The cantilever consists of a metallic signal line and a ground plane, separated by a dielectric, which determines the mechanical properties of the cantilever. The probe-cantilever interaction can be described with a lumped element circuit. (b) Cross section of the microstrip shielded cantilever. $w$ and $h$ denote the width of the signal line and the height of dielectric layer, respectively. (c) Equivalent lumped element circuit of the situation depicted in (a). (d) Distributed element circuit diagram for measuring the cantilever impedance using a balanced branchline coupler. The reflection coefficient $\Gamma$ at the cantilever tip is determined by measuring the scattering parameter $S_{41}$ of the branchline coupler. This is achieved by interference of the reflected signal from the tip with an unknown phase shift $\Delta \Phi_{2}$ with that of a balanced cancellation arm with known phase shift $\Delta \Phi_{3} . Z_{0}$ denotes the characteristic line impedance, and $\lambda$ is the signal wavelength.

line impedance $Z_{0}=50 \Omega: \Gamma=\left(Z-Z_{0}\right) /\left(Z+Z_{0}\right)$. When the cantilever is lifted far away from the sample surface, $Z$ is given by the capacitance $C_{t}$ between the tip and the cantilever ground plane [see Fig. 1(a)]. When the tip is on a sample, $Z$ is modified by contributions from the tip-sample capacitance $C_{s, t i p}$, the capacitance between the sample and the ground plane $C_{s, \text { gnd }}$, and resistive losses inside the sample, $R_{s}$. Measuring changes in $\Gamma$ by detecting phase and amplitude of the reflected signal while scanning the tip over the sample provides a quantitative image of the spatial distribution of the conducting and dielectric properties of the sample. ${ }^{5}$ Since the electric field becomes strongly enhanced at the sharp tip, these local contributions dominate the total response, thus enabling spatial resolution down to $100 \mathrm{~nm}$, i.e., three orders of magnitude below the signal wavelength.

The tip-ground plane capacitance $C_{t}$ is generally given by the size and geometry of the cantilever close to the tip, which results in values of the order of $C_{t} \sim 10^{-15} \mathrm{~F}$. Since $Z \propto 1 / i \omega C$, at gigahertz frequencies $\left(\omega=2 \pi f, f \sim 10^{9} \mathrm{~Hz}\right.$ ), the terminating impedance is large $Z \sim 10^{6} \Omega \gg Z_{0}$ and therefore the reflection coefficient becomes $\Gamma \simeq 1$. This means that most of the signal is reflected back into the 
cantilever when the tip is floating over the sample. We will refer to this part of the signal as a scattered signal because it does not carry information about the sample itself. When the tip is in contact with the sample, the desired contributions from the tip-sample interaction thus only lead to small variations on top of an otherwise large $\Gamma$, which is obviously difficult to detect. It is therefore highly desirable to minimize the scattered signal in the detector line and to become sensitive to those contributions only, which originate from the tip-sample interaction. At microwave frequencies, this problem has been addressed by making the cantilever and the tip part of a resonator ${ }^{7,8,11,12,21}$ or by adding an impedance matching circuit, which matches the open tip impedance to $Z_{0}{ }^{5}$ Both solutions create a narrow band resonance condition, which enhances the sensitivity of the circuit to changes in the tip impedance. Furthermore, a common mode cancellation loop is typically included into the microwave readout circuit, ${ }^{5,12}$ which further reduces the scattered signal level at the detector.

While at microwave frequencies such circuitry can be incorporated rather easily, this is not straight forward at $\mathrm{THz}$ frequencies because many required technologies are not readily available. In order to realize a shielded impedance microscope cantilever at $\mathrm{THz}$ frequencies, it is therefore plausible to aim for an on-chip $\mathrm{THz}$ circuit solution that can be patterned close to the tip with lithographical means. We identify the following key properties such a circuit should provide: (1) separation of the in-going and reflected signal to facilitate signal processing, (2) cancellation of the scattered signal in the detector line, (3) sensitive response when the tip is brought into contact with a dielectric or a metallic sample, and (4) short signal lines to minimize losses. In Sec. III, we will present and demonstrate a circuit that fulfills all of these requirements.

\section{BALANCED BRANCHLINE COUPLER AS ON-CHIP INTERFEROMETER}

Figure 1(d) depicts the diagram of a circuit designed to accomplish the above criteria. A key component is the balanced branchline coupler. It consists of 4 ports (labeled 1-4) that are connected through transmission line segments of a quarter wavelength $\lambda / 4$ of the aimed for measurement frequency. By properly designing the impedance of each branch of the coupler [i.e., by choosing the appropriate signal line width $w$ for a constant thickness of the dielectric layer, cf. Fig. 1(b)], one can control the transmission coefficients between the ports. The key idea of the concept we introduce here derives from analogies between a branchline coupler and an optical beam splitter: When the branch impedances $Z$ are chosen such that for two opposite branches $Z=Z_{0}(w=3.75 \mu \mathrm{m})$, while for the other two $Z=Z_{0} / \sqrt{2}(w=7.5 \mu \mathrm{m})$, an incoming signal at, for example, port 1 , is split in equal parts between ports 2 and 3 , and it acquires an additional phase shift of $-\pi / 2$ between these ports, while no signal arrives at port $4 .{ }^{22}$ Since the coupler is designed symmetrically, the signal is split in the same fashion when injected at any other port.

We can now use these properties, signal splitting and phase delay, to build an on-chip interferometer that is highly sensitive to impedance changes at the cantilever tip: We attach transmission lines of finite length $L_{2}$ and $L_{3}=L_{2}+\Delta L$ at ports 2 and 3, respectively, as shown in Fig. 1(d) (which we will refer to as arms, in analogy to an optical Michelson-interferometer). As the signal gets reflected at the end of each arm, it picks up a phase shift and gets reinjected into the coupler. For simplicity, assuming an ideal coupler with perfect isolation $^{22}$ and neglecting losses, the signal at port 4 (detector line) is then given by the sum of the reflected signals reinjected at ports 2 and 3 ,

$$
S_{41}=\frac{A}{2} e^{i\left(\Phi_{c}+\Phi_{L 2}+\Delta \Phi_{2}\right)}+\frac{A}{2} e^{i\left(\Phi_{c}+\Phi_{L 3}+\Delta \Phi_{3}\right)},
$$

where $A$ corresponds to the total signal amplitude, $\Phi_{c}=3 \pi / 2$ is the total phase accumulated in the coupler, $\Phi_{L 2,3}$ refer to the phase picked up due to the signal traveling down the respective arms, and $\Delta \Phi_{2,3}$ is the phase picked up due to reflection at the terminations of arms 2 and 3, respectively. For our purpose, it is convenient to express Eq. (1) as

$$
S_{41}=\frac{A}{2} e^{i\left(\Phi_{c}+\Phi_{L 2}\right)}\left(e^{i \Delta \Phi_{2}}+e^{i\left(\Delta \Phi_{3}+\Phi_{\Delta L}\right)}\right),
$$

which indicates that signal cancellation in the detector line is achieved for

$$
\Delta \Phi_{2}=\Delta \Phi_{3}+\Phi_{\Delta L}-\pi
$$

As shown in Fig. 1(d), in our case, arm 2 terminates in the cantilever tip, which, in a first approximation $\left(C_{t} \rightarrow 0\right)$, acts as an open termination $\left(\Delta \Phi_{2} \rightarrow-\pi\right)$ when the tip is lifted off the sample. It is therefore convenient to terminate arm 3 with a short $\left(\Delta \Phi_{3}=0\right)$ and to choose $\Phi_{\Delta L}=0$ to achieve good signal cancellation at port 4. When scanning, changes in the dielectric or metallic environment of the tip lead to a phase mismatch at the detector line due to an enhanced capacitance at the tip, according to Fig. 1(c). This results in a measurable signal that can be directly related to the phase change due to modified reflection conditions at the tip, using Eqs. (2) and (3). When the tip is landed on a fully metallic sample, $\left(1 / C_{s, t i p}+1 / C_{s, g n d}\right)^{-1} \gg C_{t}$. As can be seen from the circuit in Fig. 1(c), this corresponds to arm 2 being effectively shorted. In this case, the reflected signals will interfere constructively and the full signal is detected at port 4 . We note that in a real cantilever, $C_{t}$ is finite $\left(\Delta \Phi_{2} \gtrsim-\pi\right)$ in which case $\Delta L$ can be used as an additional phase matching parameter to achieve cancellation of the scattered signal.

In order to test this concept, we have realized a series of balanced branchline couplers on a Si substrate using the technology described by Finkel et al. ${ }^{19}$ All structures consist of $3 \mu \mathrm{m}$ thin $\mathrm{SiN}_{x}$ $\left(\varepsilon_{r}=5.9\right)$, serving as a MS dielectric and $2 / 300 \mathrm{~nm} \mathrm{Ti} / \mathrm{Au}$ as a ground plane and a strip line. As $\mathrm{THz}$ source and detector, we use a vector network analyzer together with frequency multipliers that cover the WR-03 band (220-325 GHz) and a GSG landing probe setup (for details, see Finkel et al. ${ }^{19}$ ). Note, however, that our concept is also compatible with other $\mathrm{THz}$ sources and detectors, for instance photomixers. ${ }^{23,24}$ We first demonstrate conceptually the basic idea. For this, we have fabricated two samples (devices $\mathrm{A}$ and B) for which $\Delta L=0$ and realize two different arm terminations open/short and open/open at ports 2 and 3, respectively. An optical image of device A is shown in Fig. 2(a). The signal is launched and picked up from the circuit via the ports labeled 1 and 4 in Fig. 2(a), which consist of coplanar waveguide type fixtures $^{19}$ (not visible) that enable coupling of $\mathrm{THz}$ signals into the circuit with the landing probes. The $\lambda / 4$-branches of the coupler have a length of $130 \mu \mathrm{m}$, corresponding to a branchline coupler 
a)

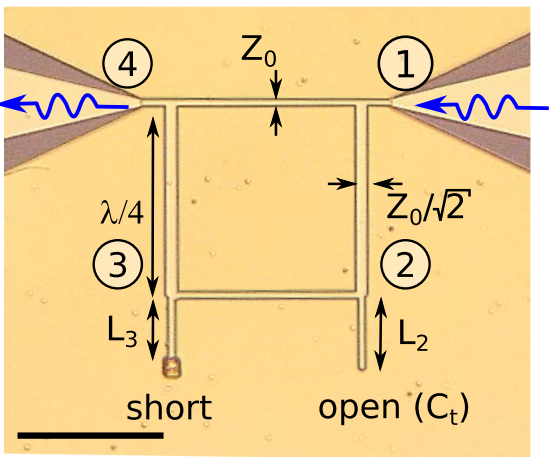

b)

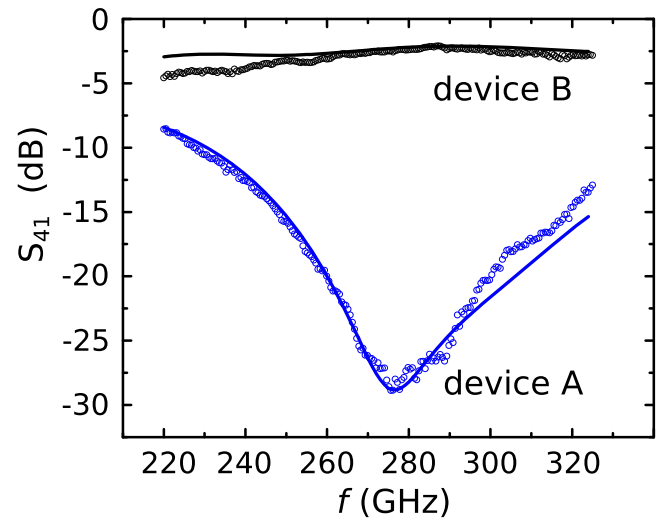

FIG. 2. (a) Optical image of the on-chip interferometer (device A), containing a balanced branchline coupler with branch lengths $\lambda / 4$. The ports of the coupler are denoted as $1-4$. At a distance $L_{2}$ and $L_{3}$ from ports 2 and 3 , the transmission lines terminate in an open and short circuit, respectively. The signal (indicated with blue arrows) is injected at port 1 and detected at port 4 . The scale bar corresponds to $100 \mu \mathrm{m}$. (b) S-parameter magnitude of a transmission measurement (symbols) and the corresponding analytic calculation (solid line) of devices A (blue) and B (black). $L_{2}=L_{3}=49 \mu \mathrm{m}$.

center frequency of $f_{c}=270 \mathrm{GHz}$. For the arm lengths, we choose $L_{2}=L_{3}=49 \mu \mathrm{m}$.

Figure 2(b) shows the measured scattering parameter $S_{41}$ obtained for devices A and B (blue and black symbols, respectively). As expected, for device A, we observe a low transmission $(\sim-30 \mathrm{~dB})$ between ports 1 and 4 with a minimum at $f=280 \mathrm{GHz}$, which is close to the branchline coupler's center frequency $f_{c}=270 \mathrm{GHz}$. For device $B$, both arms terminate in an open, i.e., $\Delta \Phi_{2}=\Delta \Phi_{3}$. As a result, constructive interference leads to a high transmission $(\sim-5 \mathrm{~dB})$ over the full frequency range.

Next, we demonstrate how, owing to the sharp interferometer cancellation conditions, the circuit is highly sensitive to contributions from $\Phi_{\Delta L}$. Figure 3 shows the measured $S_{41}$ parameter obtained from a series of devices for which we have varied $L_{3}=49 \mu \mathrm{m}+\Delta L$ by $\Delta L=(1,0,-1, \ldots,-5) \mu \mathrm{m}$, while leaving $L_{2}=49 \mu \mathrm{m}$ fixed. This leads to a small phase imbalance for the

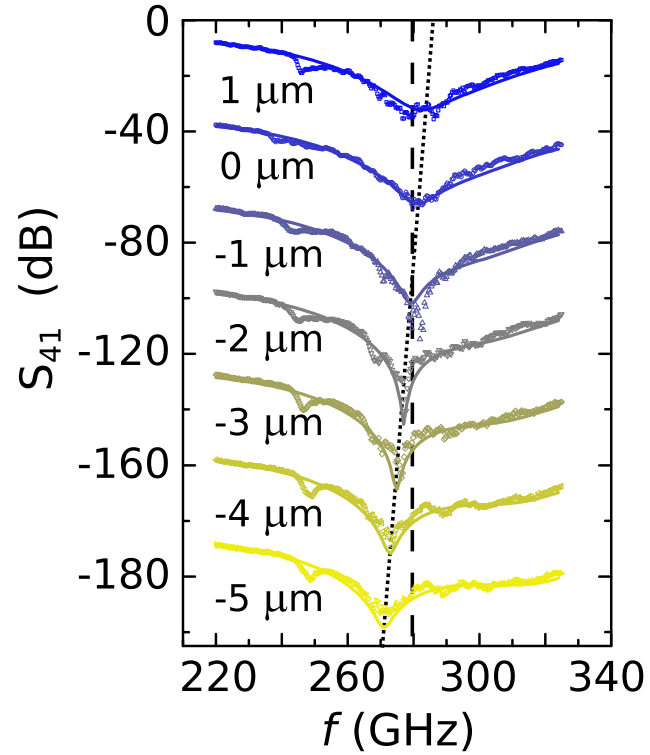

FIG. 3. Transmission measurements (symbols) and calculation (solid line) for a set of open/short circuits with $L_{3}=L_{2}+\Delta L$ and $\Delta L=+1,0, \ldots,-5 \mu \mathrm{m}$ and $L_{2}=49 \mu \mathrm{m}$. The curves are offset by $-30 \mathrm{~dB}$ for clarity.

signal paths along arms 2 and 3. The experimental data reveal that indeed the position of the dip in frequency as well as its depth sensitively depend on $\Delta L$ (dotted line). In Fig. 4(a), we have extracted magnitude and phase (symbols) for each $\Delta L$ at fixed frequency $f=280 \mathrm{GHz}$ (dashed line in Fig. 3). The data show that signal cancellation improves for small $\Delta L$ with an optimal configuration at $\Delta L=-1 \mu \mathrm{m}$. For even larger length difference, it levels off. As discussed above, this behavior reflects the termination of arm 2 with a finite capacitance, leading to phase shift slightly different from $-\pi$, which gets compensated for by a slightly shorter $L_{3}$. This has been confirmed quantitatively within a textbook analytical model of the circuit $^{22}$ (for details, see the Appendix and the supplementary material) that nicely reproduces all of our experimental data consistently [solid lines in Figs. 3 and 4(a)]. In addition to a small dissipative contribution in the via, $R_{\text {short }}=1.6 \Omega$, we have taken into account a finite terminating capacitance $C_{t}=0.163 \mathrm{fF}$, consistent with a standard text book approximation for an open MS line (see the Appendix).

We can further use the analytical model to analyze theoretically the circuit's response to a load capacitance $C_{\text {load }}$ connected in parallel to $C_{t}$, representing a sample in a scanning probe experiment [cf. lumped element diagram in Fig. 1(c)]. The resulting amplitude and phase are plotted in Fig. 4(a) as dashed lines. The corresponding $C_{\text {load }}$ is given in the top axis. As expected, this yields a fairly similar behavior as a variation of $\Delta L$. Figure 4 (b) plots the data as in-phase (I) and quadrature (Q) amplitudes, for a variation of $\Delta L$ (bottom axis, solid lines) and $C_{\text {load }}$ (top axis, dashed lines), respectively. In this representation, $I$ can be directly related to dissipative contributions to the signal, while $Q$ represents the imaginary part of the reflection coefficient, which is related to capacitive (and, in principle, also inductive) contributions. This is consistent with the observed linear behavior of $Q$ and a constant $I$. From these plots, we 
a)

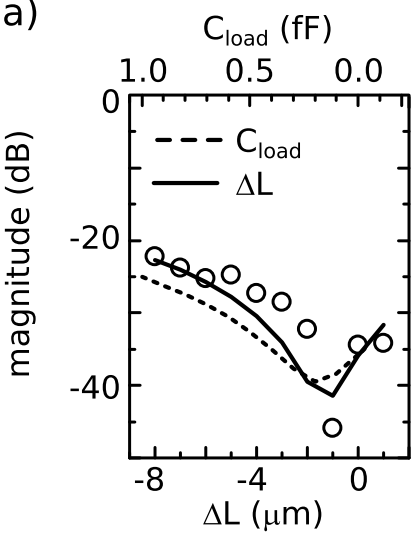

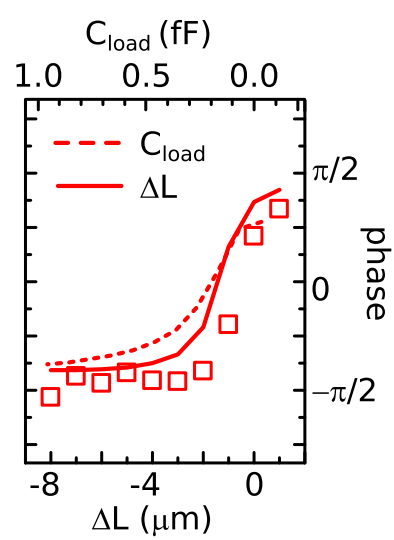

b)

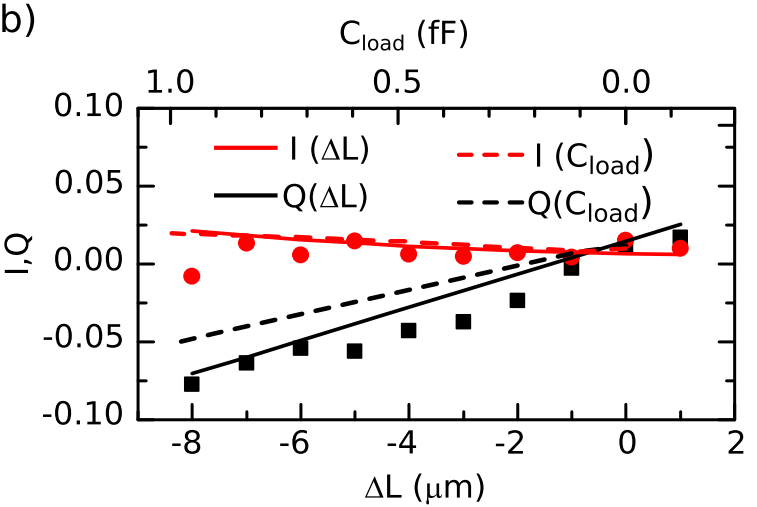

FIG. 4. (a) Magnitude (left) and phase (right) as a function of $\Delta L$ (bottom axis). Symbols: measurements; solid line: calculation; dashed line and top axis: calculated phase and magnitude for $L_{2}=L_{3}=49 \mu \mathrm{m}$ and with varying $C_{\text {load }}$ connected in parallel to the termination of interferometer arm 2. (b) In-phase (I, red) and quadrature $(Q$, black) representation of the measured (circles and squares) and calculated (solid and dashed line) response vs $\Delta L$. Dashed lines and top axis: calculated I (red) and $Q$ (black) as a function of $C_{\text {load }}$.

estimate our circuit to be sensitive to a capacitance change smaller than $0.25 \mathrm{fF}$.

\section{CANTILEVER IMPLEMENTATION}

We will now describe how this detection scheme can be implemented and used in a scanning probe cantilever to detect impedance changes at the probe tip. Figure 5(b) shows an optical microscope image of the shielded cantilever containing the $\mathrm{THz}$ circuit, patterned on its top side. The signal in and signal out lines [corresponding to ports 1 and 4 in Fig. 2(a)] are connected via landing probes with the source and detector (not visible). Since the dimensions of the cantilever ( $300 \mu \mathrm{m}$ long and $75 \mu \mathrm{m}$ wide) are too small to host a circuit as shown in Fig. 2(a), we have redesigned the branchline coupler such that the cross-branches are now folded inwards to fit the lateral dimensions of the cantilever. This slightly modifies the coupler properties. However, it does not change its basic functionality. As discussed previously for the branchline coupler devices, one of the interferometer arms terminates in a short. The other one, previously terminating in an open, is now connected to the tip. We will keep the notation of the arms as introduced above, referring to the arm terminating in the tip as arm 2 with length $L_{2}$ and to the arm terminating in a short to ground as arm 3 with length $L_{3}$. In order to balance the coupler such that scattered signal cancellation is achieved, we have to take into account the finite capacitance of the open tip [ $C_{t} \sim 2 \mathrm{fF}$, obtained from finite element (FE) simulations] and adjust $L_{3}$ by $\Delta L$ accordingly. However, due to the folded geometry of the coupler and a resulting unwanted cross coupling between the branches, significant leakage currents within the coupler result in a nontrivial relation between $\Delta L$ and signal cancellation at the detector line. Therefore, we use FE simulations to empirically determine a well-balanced configuration for the given $C_{t}$, for which we obtain $L_{2}=44 \mu \mathrm{m}$ and $L_{3}=54 \mu \mathrm{m}$, i.e., $\Delta L=8 \mu \mathrm{m}$.

\section{A. Fabrication}

In Fig. 5(a), the fabrication flow for the cantilever is sketched. In step (1), a pyramid shaped pit ( $5 \mu \mathrm{m}$ deep) is etched into the $\mathrm{Si}$ wafer using $\mathrm{KOH}$ etching. This defines the position and shape of the tip. In step (2), we deposit $(10+300) \mathrm{nm} \mathrm{Ti} / \mathrm{Au}$ that serves as a ground plane. During this step also, the pit is filled with a $\mathrm{Ti} / \mathrm{Au}$ layer, which will become the metallic tip. The area around the pit is protected with an optical mask. The wafer is then (3) covered with $3 \mu \mathrm{m}$ of PECVD SiNx that is subsequently etched with a Bosch process to define the geometry of the cantilever, $300 \mu \mathrm{m}$ long and $75 \mu \mathrm{m}$ wide. In a separate step (4), $5 \times 5 \mu \mathrm{m}^{2}$ sized vias are etched into the $\mathrm{SiN}_{x}$ layer. These vias will serve as an electrical connection between the MS top layer and the ground plane (to form a short) or with the cantilever tip, respectively. After a cleaning step, we pattern the strip lines with electron beam lithography and lift off techniques (5) and, in a separate step, connection of the via is established through angled deposition of Au. We use $(2+300) \mathrm{nm} \mathrm{Ti} / \mathrm{Au}$ bilayers. This step concludes the patterning of the transmission lines on the cantilever. Next, we release the cantilevers (6). In order to avoid exposure of the strip lines to chemicals, we protect the surface of the wafer by gluing a Sp wafer on top of it with "black wax" (Apiezon W100). We take particular care that no air bubbles remain in the wax to ensure a complete and efficient protection. The release step is prepared by patterning a $\mathrm{SiN}_{x}$ mask on the backside of the wafer that contains windows at those positions where the cantilevers have been patterned on the front side of the wafer. The two wafers are then subjected to a $\mathrm{KOH}$ etch that etches through the windows on the backside of the wafer until the $\mathrm{Ti} / \mathrm{Au}$ ground layer is reached. At this point, the cantilever gets released from the Si wafer. Note, however, that on its front side, it is still glued to the protection wafer. When the $\mathrm{KOH}$ etch is complete, the wafer is carefully immersed in toluene to dissolve the black wax and to fully release the cantilever chips. Subsequently, the cantilever is mounted on the landing probe setup.

\section{B. Experimental results}

The measured $\mathrm{THz}$ response of the cantilever is shown in Fig. 5(c). We clearly observe a dip in transmission $(\sim-30 \mathrm{~dB})$, indicating a suppression of the scattered signal that gets reflected from the open tip into the detector line. We note that compared to the previously discussed branchline couplers without the tip (Fig. 2), the position of the dip is slightly shifted toward lower frequencies 
a)

1. $\mathrm{Si}$

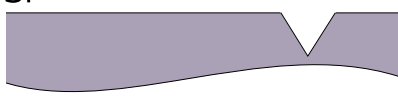

2. $\mathrm{Ti} / \mathrm{Au}$

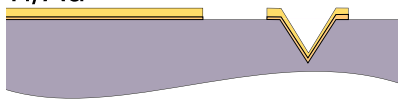

3. SiNx

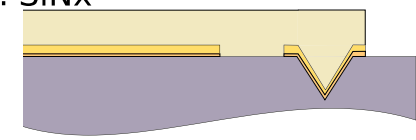

4.

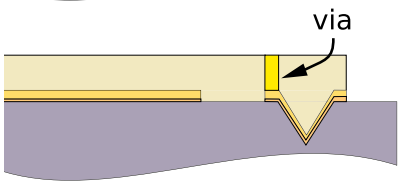

5. $\mathrm{Ti} / \mathrm{Au}$

6.
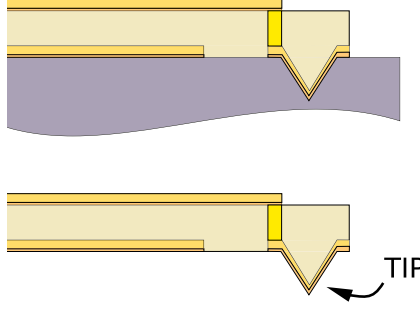

b)

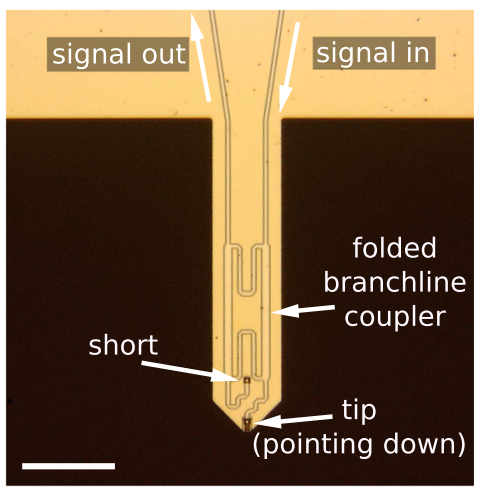

c)

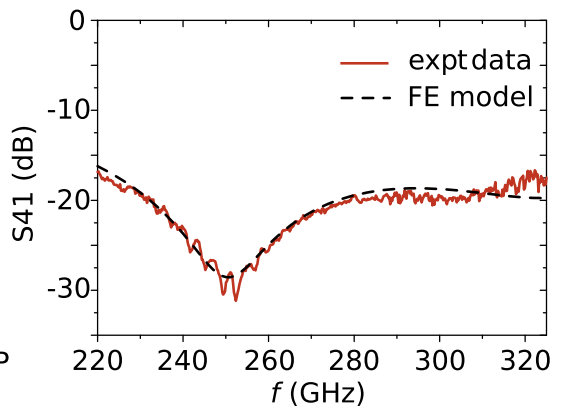

FIG. 5. (a) Main fabrication steps of the THz cantilever. (b) Optical image of the final, released cantilever. The scale bar corresponds to $75 \mu \mathrm{m}$. Signal in and signal out denote the transmission lines connected to $\mathrm{THz}$ source and detector, respectively. The folded hybrid coupler patterned on the back of the cantilever is indicated. One arm of the coupler terminates in a short circuit; the other one terminates in the tip. (c) Transmission amplitude from signal in to signal out. The transmission dip at $250 \mathrm{GHz}$ indicates cancellation of the signal reflected from the open tip. The dashed curve shows the result obtained from finite element (FE) modeling. $(f=250 \mathrm{GHz})$. This is most likely a result of the folded geometry of the branchline coupler, consistent with a cross-capacitive coupling between neighboring parts of the circuit. Moreover, the frequency shift as well as the relatively low transmission at higher and lower frequencies suggests that the terminating capacitance of the tip slightly differs from the assumed value $\left(C_{t}=2 \mathrm{fF}\right)$ such that the chosen $\Delta L=8 \mu \mathrm{m}$ turns out to be not yet the best match. The FE model for the $\mathrm{THz}$ response (dashed line) yields good agreement with the experiment if we assume $C_{t}=2.9 \mathrm{fF}$ and $R_{\text {short }}=5 \Omega$.

Our cantilever can be used to detect changes in the tip impedance when landed on a dielectric or metallic sample. This is demonstrated in Fig. 6. We have mounted the cantilever on the landing probe setup and we get the tip in contact with different materials, approached from below via a mechanical height control. Figure 6(a) compares the measured response for the tip floating near the sample surface (red) and landed on 3 different materials, Au (green), $\mathrm{Si}$ (black), and $\mathrm{SrTiO}_{3}$ (blue). We clearly observe distinct responses for each material. When brought into contact with a dielectric ( $\mathrm{Si}$, $\mathcal{E}_{r}=11.9$ and $\mathrm{SrTiO}_{3}, \varepsilon_{r}=300$ ), the dip shifts toward lower frequencies by $\Delta f_{(\mathrm{Si})}=2 \mathrm{GHz}$ for $\mathrm{Si}$ and $\Delta f_{(\mathrm{STO})}=10 \mathrm{GHz}$ for $\mathrm{SrTiO}_{3}$. Notably, the overall line shape remains fairly similar. In contrast, upon contact with highly conductive Au $(\rho=2 \mu \Omega \mathrm{cm})$, the dip vanishes and transmission is high over the full frequency range, as expected for a shorted tip.

\section{Discussion}

In order to quantitatively understand the cantilever response, we use FE modeling of the full circuit and we include a load capacitance $C_{\text {load }}$ in parallel to $C_{t}$ to take into account contributions from the sample materials [cf. Fig. 1(a), neglecting Ohmic dissipation in the sample, $\left.R_{s}=0\right]$. We find that the curves can be reproduced very well if we use $C_{\mathrm{Si}}=0.25 \mathrm{fF}, C_{S T O}=0.75 \mathrm{fF}$, and $C_{\mathrm{Au}}=15 \mathrm{fF}$ as the only adjustable parameter for each material. In Fig. 6(b), we compare $I$ and $Q$ of the FE response for various $C_{\text {load }}$ with the experimental values obtained at $f=260 \mathrm{GHz}$ for each sample. Since the response of the folded branchline coupler connected to the tip is slightly off resonance $(\sim 250 \mathrm{GHz})$, we do not expect a simple linear behavior as for the branchline coupler without the tip discussed previously. Figure 6(b) shows that the response becomes more sensitive, i.e., the slope of the curves for $I$ and $Q$ becomes steep, for larger $C_{\text {load }}(\sim 10 \mathrm{fF})$. Since this is in the range of capacitance, which we obtained for the Au sample, this indicates that our $\mathrm{THz}$ cantilever becomes more sensitive for metallic samples. In contrast to shielded microwave cantilevers, where sensitivity is highest around the metal-insulator transition, ${ }^{5}$ for our cantilever, the working point is shifted toward samples with higher conductivity. It may thus be used to detect electronic variations at high frequencies within a metal or even in superconductors in future scanning experiments.

Our $\mathrm{THz}$ cantilever is also compatible with AFM topography imaging. This is shown in Fig. 6(c) where a topography image of a scratched Si wafer surface is displayed, obtained using a $\mathrm{THz}$ cantilever mounted on a commercial Asylum Cypher AFM with a laser deflection readout. Using a deconvoluting tip geometry modeling algorithm (Gwyddion blind tip estimation algorithm ${ }^{25}$ ), we estimate the tip apex to be $\approx 100 \mathrm{~nm}$. An SEM image of a cantilever tip is shown in Fig. 6(d). 
a)

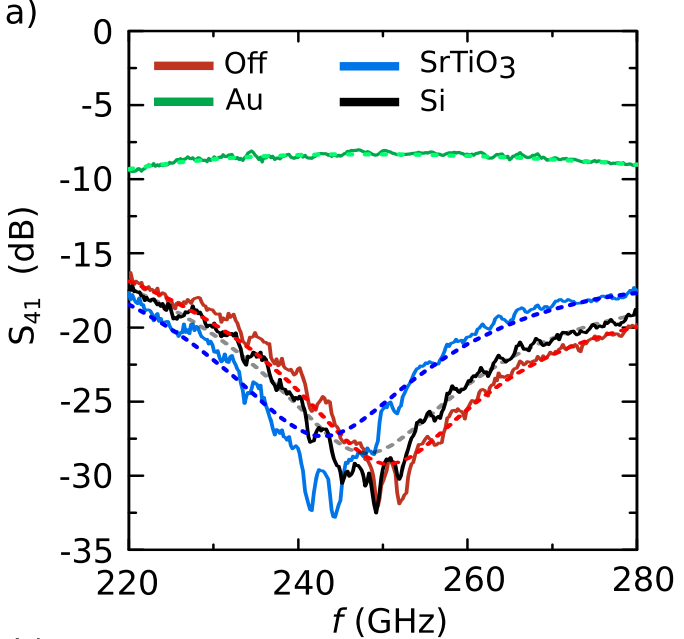

b)

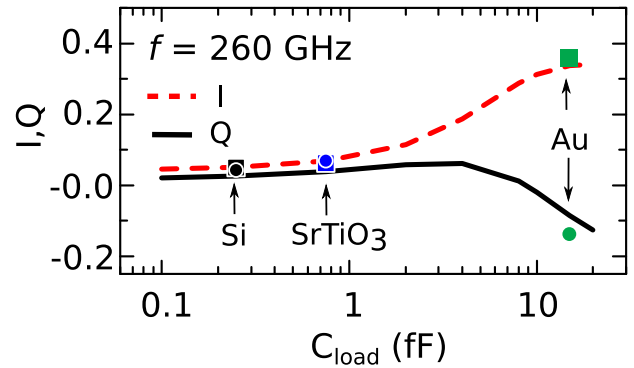

C)

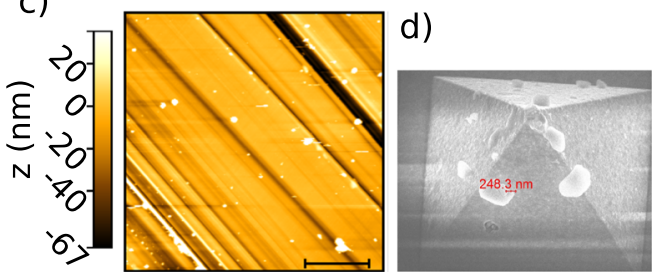

FIG. 6. (a) THz response of the cantilever when open (red) and landed on $\mathrm{Si}$ (black), $\mathrm{SrTiO}_{3}$ (blue), and $\mathrm{Au}$ (green) samples. Solid lines correspond to measurements and dashed lines indicate finite element (FE) simulations with the following parameters: $R_{\text {via }}=5 \Omega$, tip capacitance: $C_{t}=2.9 \mathrm{fF} ; L_{2}=44 \mu \mathrm{m}, L_{3}=52 \mu \mathrm{m}$, conductivity signal line and ground plane: $\sigma_{\text {au }}=19.2 \times 10^{-6} \mathrm{~S} / \mathrm{m}, \varepsilon_{r}\left(\mathrm{SiN}_{x}\right)=5.9$. $C_{\text {load }}$ [corresponding to $\left(1 / C_{s, t i p}+1 / C_{s, g n d}\right)^{-1}$ in Fig. $1, R_{s}=0$ ] for Off: $0 \mathrm{fF}$; Si: 0.25 fF; $\mathrm{SrTiO}_{3}: 0.75 \mathrm{fF} ; \mathrm{Au}: 15 \mathrm{fF}$. (b) I (red dashed line) and $\mathrm{Q}$ (black solid line) of the cantilever response at $260 \mathrm{GHz}$ for different $C_{\text {load }}$ obtained from FE simulations. Symbols: experimental data extracted from (a). (c) $20 \mu \mathrm{m} \times 20 \mu \mathrm{m}$ topography image of a scratched Si wafer, obtained with a cantilever similar to the one used in (a) utilizing the beam deflection mode in a commercial AFM. (d) SEM image of the cantilever tip.

Finally, we like to point out some aspects that we aim to improve for future $\mathrm{THz}$ cantilever generations. First, even though our fabrication technique provides useful devices, the current yield is rather low $(\approx 10 \%)$. This is mostly related to the use of the black wax, which is needed to protect the $\mathrm{THz}$ circuitry from chemicals during the release step, but which also induces mechanical stress, resulting in loss of a large number of cantilevers. Second, in its current design, the substrate, which serves as a handling wafer, faces in the same direction as the tip. This limits the surface region on the sample that can be reached by the cantilever to approximate the cantilever length $(\sim 300 \mu \mathrm{m})$. In order to lift this constraint, developing a flip-chip technology may provide the most suitable means to bond a handling wafer to the top side of the cantilever chip. At the same time, however, it will be important to maintain access to the circuitry with landing probes. Third, our experiments have shown that due to the folded geometry of the balanced branchline coupler, the circuit response deviates slightly from that of the unfolded geometry tested on a substrate [Fig. 5(b) in comparison to Fig. 2(a)]. Therefore, FE simulations are required for a quantitative analysis of the measurement signal, while a simple analytical equation would be more desirable. This may motivate a redesign of the cantilever such that it can host the balanced coupler without the need to modify its layout. In this case, the measurements could be modeled within a textbook analytical description, which will strongly facilitate a quantitative interpretation of the measurement signal.

\section{CONCLUSION}

We have presented a shielded $\mathrm{THz}$ probe suitable for impedance microscopy in the sub-millimeter band $(0.3 \mathrm{THz})$. As a key challenge for the realization of such a device, we have identified the necessity to carry out common mode cancellation and impedance matching at $\mathrm{THz}$ frequencies close to the cantilever tip in order to enable sensitive detection of small changes of the tip impedance. We have addressed these challenges by developing an on-chip circuit that can be patterned on the cantilever, which comprises a balanced branchline coupler. The coupler functions as an on-chip interferometer and in this manner achieves the required common mode suppression as well as high sensitivity to small impedance changes. To demonstrate the basic functionality of this concept, we have realized a set of devices on substrates and we have characterized their response at $\mathrm{THz}$ frequencies. The results can be directly modeled within an analytical model of the circuit. Furthermore, a fabrication technology has been developed that allows for patterning the circuit on a free standing cantilever including the tip. When the released cantilever is landed on different dielectric $\left(\mathrm{Si}, \mathrm{SrTiO}_{3}\right)$ and metallic ( $\mathrm{Au}$ ) samples, we observe distinct $\mathrm{THz}$ responses, which enable us to determine the corresponding capacitive load at the cantilever tip using finite element modeling. Our cantilever removes several critical technological challenges toward scanning impedance microscopy at $\mathrm{THz}$ frequencies.

\section{SUPPLEMENTARY MATERIAL}

The supplementary material includes a derivation of the analytical model of the balanced hybrid coupler and a detailed recipe for the fabrication of the $\mathrm{THz}$ cantilevers.

\section{ACKNOWLEDGMENTS}

We would like to thank Carmine de Martino and Luca Galatro for help with the $\mathrm{THz}$ characterization setup and David Thoen 
for support during the fabrication process development. We further acknowledge scientific discussions with Andrea Neto, Nuria Llombart, Akira Endo, Jochem Baselmans, Ronald Hesper, and Iván Cámara Mayorga. HT acknowledges hospitality support from Andrea Caviglia at TU Delft. This research has been funded by the European Research Council Advanced Grant No. 339306 (METIQUM).

\section{APPENDIX: ANALYTICAL MODEL FOR THE BALANCED BRANCHLINE COUPLER}

In order to compute the response of the balanced hybrid coupler, we describe the signal evolution in the coupler in terms of forward and backward traveling waves in the transmission lines and the reflection coefficients $\Gamma_{\mathrm{ms}}$ and $\Gamma_{\mathrm{s}}$ at the open and shorted transmission line, respectively. Using Kirchoff's rules for the voltage and current at each node of the hybrid, we can construct a system of equations that allows us to determine the voltage measured at the detector line at port 4 upon signal injection at port 1. (The full set of equations is provided in the supplementary material.) To describe wave propagation along each transmission line segment with length $L$ and impedance $Z$, we use the frequency dependent wave propagation factor $e^{\gamma L}$ and

$$
\begin{gathered}
\gamma=\alpha \frac{Z}{Z_{0}}+i \beta, \\
\alpha=\left(1.1 f \times 10^{-9}+86.9\right) \mathrm{Np} / \mathrm{m}, \\
\beta=\frac{2 \pi}{c} f \sqrt{\varepsilon_{\mathrm{eff}}},
\end{gathered}
$$

with $Z_{0}=55.5 \Omega, c=3 \times 10^{8} \mathrm{~m}(\mathrm{~s})^{-1}, \varepsilon_{\text {eff }}=4.47$, and $\alpha$ extracted from a direct measurement of a $130 \mu \mathrm{m}$ transmission line. For the low impedance lines, we have used $Z=Z_{l}=37 \Omega$. The branch lengths of the coupler are $L=130 \mu \mathrm{m}$.

To calculate the open terminating capacitance $C_{t}$ of the open MS line, we have used

$$
\begin{gathered}
C_{t}=G \frac{\sqrt{\varepsilon_{e f f}}}{c Z_{0}}, \\
G=\frac{\xi_{1} \xi_{3} \xi_{5} h}{\xi_{4}},
\end{gathered}
$$

together with the following closed form expression:

$$
\begin{aligned}
& \xi_{1}=0.434907 \frac{\left(\varepsilon_{e f f}^{0.81}+0.26(w / h)^{0.8544}+0.236\right)}{\left(\varepsilon_{e f f}^{0.81}-0.189(w / h)^{0.8544}+0.87\right)}, \\
& \xi_{2}=1+\frac{(w / h)^{0.371}}{2.35 \varepsilon_{r}+1}, \\
& \xi_{3}=1+\frac{0.5274 \tan ^{-1}\left[0.084(w / h)^{1.9413 / \xi_{2}}\right]}{\varepsilon_{e f f}^{0.9236}}, \\
& \xi_{4}=1+0.037 \tan ^{-1}\left[0.067(w / h)^{1.456}\right]\left(6-5 \exp \left(0.036\left(1-\varepsilon_{r}\right)\right)\right), \\
& \xi_{5}=1-0.218 \exp (-7.5(w / h)),
\end{aligned}
$$

for which we have used the strip line width $w=3.75 \mu \mathrm{m}$, dielectric thickness $h=3 \mu \mathrm{m}, \varepsilon_{\text {eff }}=4.47$, PECVD SiN $x$ dielectric constant $\varepsilon_{r}=5.9,{ }^{19}$ characteristic impedance $Z_{0}=55.5 \Omega$, and the vacuum speed of light $c=3 \times 10^{8} \mathrm{~m} / \mathrm{s}$.

\section{REFERENCES}

${ }^{1}$ D. N. Basov, R. D. Averitt, D. Van der Marel, M. Dressel, and K. Haule, "Electrodynamics of correlated electron materials," Rev. Mod. Phys. 83, 471 (2011).

${ }^{2}$ M. M. Qazilbash, M. Brehm, B.-G. Chae, P.-C. Ho, G. O. Andreev, B.-J. Kim, S. J. Yun, A. Balatsky, M. Maple, F. Keilmann et al., "Mott transition in $\mathrm{VO}_{2}$ revealed by infrared spectroscopy and nano-imaging," Science 318, 1750-1753 (2007).

${ }^{3}$ M. Liu, A. J. Sternbach, and D. Basov, "Nanoscale electrodynamics of strongly correlated quantum materials,” Rep. Prog. Phys. 80, 014501 (2016).

${ }^{4}$ A. J. Huber, F. Keilmann, J. Wittborn, J. Aizpurua, and R. Hillenbrand, “Terahertz near-field nanoscopy of mobile carriers in single semiconductor nanodevices," Nano Lett. 8, 3766-3770 (2008).

${ }^{5}$ K. Lai, M. Nakamura, W. Kundhikanjana, M. Kawasaki, Y. Tokura, M. A. Kelly, and Z.-X. Shen, "Mesoscopic percolating resistance network in a strained manganite thin film," Science 329, 190-193 (2010).

${ }^{6}$ P. J. de Visser, R. Chua, J. O. Island, M. Finkel, A. J. Katan, H. Thierschmann, H. S. van der Zant, and T. M. Klapwijk, "Spatial conductivity mapping of unprotected and capped black phosphorus using microwave microscopy," 2D Mater. 3, 021002 (2016).

${ }^{7}$ G. Gramse, A. Kölker, T. Lim, T. J. Stock, H. Solanki, S. R. Schofield, E. Brinciotti, G. Aeppli, F. Kienberger, and N. J. Curson, "Nondestructive imaging of atomically thin nanostructures buried in silicon," Sci. Adv. 3, e1602586 (2017). ${ }^{8}$ S. M. Anlage, V. V. Talanov, and A. R. Schwartz, "Principles of near-field microwave microscopy," in Scanning Probe Microscopy (Springer, 2007), pp. 215-253.

${ }^{9}$ D. Steinhauer, C. Vlahacos, S. Dutta, B. Feenstra, F. Wellstood, and S. M. Anlage, "Quantitative imaging of sheet resistance with a scanning near-field microwave microscope,” Appl. Phys. Lett. 72, 861-863 (1998).

${ }^{10}$ A. Buchter, J. Hoffmann, A. Delvallée, E. Brinciotti, D. Hapiuk, C. Licitra, K. Louarn, A. Arnoult, G. Almuneau, F. Piquemal et al., "Scanning microwave microscopy applied to semiconducting GaAs structures," Rev. Sci. Instrum. 89, 023704 (2018).

${ }^{11}$ A. Tselev, S. M. Anlage, Z. Ma, and J. Melngailis, "Broadband dielectric microwave microscopy on micron length scales," Rev. Sci. Instrum. 78, 044701 (2007).

${ }^{12}$ H. Huber, I. Humer, M. Hochleitner, M. Fenner, M. Moertelmaier, C. Rankl, A. Imtiaz, T. Wallis, H. Tanbakuchi, P. Hinterdorfer et al., "Calibrated nanoscale dopant profiling using a scanning microwave microscope," J. Appl. Phys. 111, 014301 (2012).

${ }^{13}$ K. Akiyama, A. Alberdi, W. Alef, K. Asada, R. Azulay, A.-K. Baczko, D. Ball, M. Baloković, J. Barrett, D. Bintley et al., "First M87 event horizon telescope results. II. Array and instrumentation,” Astrophys. J., Lett. 875, L2 (2019).

${ }^{14}$ P. H. Siegel, “Terahertz technology," IEEE Trans. Microwave Theory Tech. 50, 910-928 (2002)

${ }^{15}$ S. Dordevic and D. Basov, "Electrodynamics of correlated electron matter," Ann. Phys. 15, 545-570 (2006).

${ }^{16}$ C. Liewald, S. Mastel, J. Hesler, A. J. Huber, R. Hillenbrand, and F. Keilmann, “All-electronic terahertz nanoscopy," Optica 5, 159-163 (2018).

${ }^{17}$ M. Westig, K. Jacobs, J. Stutzki, M. Schultz, M. Justen, and C. Honingh, "Balanced superconductor-insulator-superconductor mixer on a $9 \mu \mathrm{m}$ silicon membrane," Supercond. Sci. Technol. 24, 085012 (2011).

${ }^{18} \mathrm{~J}$. Zmuidzinas and P. L. Richards, "Superconducting detectors and mixers for millimeter and submillimeter astrophysics," Proc. IEEE 92, 1597-1616 (2004).

${ }^{19}$ M. Finkel, H. Thierschmann, L. Galatro, A. J. Katan, D. J. Thoen, P. J. de Visser, M. Spirito, and T. M. Klapwijk, "Performance of THz components based on microstrip PECVD SiN $\mathrm{x}_{\mathrm{x}}$ technology," IEEE Trans. Terahertz Sci. Technol. 7, 765-771 (2017).

${ }^{20}$ K. Lai, W. Kundhikanjana, M. A. Kelly, and Z.-X. Shen, "Nanoscale microwave microscopy using shielded cantilever probes,” Appl. Nanosci. 1, 13-18 (2011). 
${ }^{21}$ Y.-T. Cui, E. Y. Ma, and Z.-X. Shen, "Quartz tuning fork based microwave impedance microscopy,” Rev. Sci. Instrum. 87, 063711 (2016).

${ }^{22}$ D. M. Pozar, Microwave Engineering (John Wiley \& Sons, 2009).

${ }^{23}$ I. C. Mayorga, A. Schmitz, T. Klein, C. Leinz, and R. Gusten, "First in-field application of a full photonic local oscillator to terahertz astronomy," IEEE Trans. Terahertz Sci. Technol. 2, 393-399 (2012).
${ }^{24}$ M. P. Westig, H. Thierschmann, A. J. Katan, M. Finkel, and T. M. Klapwijk, "Analysis of a single-mode waveguide at subterahertz frequencies as a communication channel," e-print arXiv:1909.08976 (2019).

${ }^{25}$ D. Nečas and P. Klapetek, "Gwyddion: An open-source software for SPM data analysis,” Open Phys. 10, 181-188 (2012). 